\title{
Nuclear Medicine Instrumentation
}

\author{
J. Prekeges
}

Sudbury, MA: Jones and Bartlett Publishers, 2011, \$76.95

This textbook, which has a copyright date of 2011, was published and released in early 2010 by Jones and Bartlett. The author, Jennifer Prekeges, spent more than 9 years putting the text together. She has done a wonderful job of compiling a number of varied nuclear medicine instrument references into a single comprehensive text. The organization of the book, going from small instruments to $\gamma$-cameras to SPECT, PET, and CT as it relates to nuclear medicine, carries the reader on a journey from simple to more complex instruments and how the technology works. The appendices give the reader basic background information on atomic structure, atomic interactions with matter, electronics, film and film processing, computers, and collimator mathematics. In chapter 1 , the reader is first introduced to gas-filled detectors, with an excellent explanation of the difference between current and pulse-mode detectors. Chapter 2 begins with an explanation of scintillation detectors, which are the basis for $\gamma$-cameras, and then explains in detail photomultiplier tubes, single-channel analyzers, multichannel analyzers, quality control, and full width at half maximum. Chapter 3 explains handheld semiconductor detectors, focusing on their properties, their differences from photomultiplier tubes for radiation detection, and their practical applications in surgery, tumor localization, and sentinel lymph node detection. The chapter ends with quality control and proper handling of these detectors. Chapter 4 covers factors relating to radiation measurement, such as counting statistics, dead-time loss, energy spectra, photo peaks, scatter radiation, and detector efficiency.

Chapter 5 introduces a 5-chapter section devoted to the $\gamma$-camera. A historical overview includes an explanation of the Anger positioning logic and camera components such as the collimator, crystal, photomultiplier tubes, and pulse height analyzers. This introduction is followed by an examination of the components of modern $\gamma$-cameras and associated aspects such as sources of nonuniformity, energy and linearity corrections, automatic tuning, and other means of optimizing $\gamma$-camera performance. The chapter ends with a brief mention of organspecific cameras such as those that image the heart and breast. Chapter 6 covers image digitization and display, looking first at analog imaging techniques and then at

COPYRIGHT (C) 2010 by the Society of Nuclear Medicine, Inc. DOI: $10.2967 /$ jnmt.110.081554 digital. This look includes the acquisition parameters of digital images and display characteristics. PACS and digital imaging and communications in medicine are briefly mentioned here and then are expanded on in appendix D. Chapter 7 covers collimators, including their manufacture process, and parameters such as hole diameter, length, and septal thickness. The association between distance and collimator type is also addressed. Collimator selection factors are covered, along with a brief explanation of different collimator types. Chapter 8 looks at image characteristics and performance measures in planar imaging. Factors affecting image formation, including background radiation, system noise, third-dimension superposition, resolution loss with distance, photon attenuation, scatter radiation, and patient motion, are discussed. The author then covers other aspects of image formation, including contrast, uniformity, sensitivity, count rate, spectral resolution, bar phantoms, the line spread function, the modulation transfer function, and the relationship of all of these to the resolution capabilities of the camera. The effects of the acquisition itself are explored, including parameters such as count rate, collimator choice, matrix selection, energy window setup, and object-to-collimator distance. Chapter 9 covers departmental quality assurance and the quality control of $\gamma$-cameras; this chapter starts with a nice explanation of the difference between quality assurance and quality control-terms that often are used interchangeably in clinical settings. Several examples of quality control problems are illustrated, and the chapter ends by addressing 19 common troubleshooting issues.

Chapter 10 begins a 4 -chapter section on SPECT with a basic explanation of the SPECT process (which historically preceded $\mathrm{CT}$ ) and analytic reconstruction, including unfiltered backprojection, Nyquist frequency, iterative reconstruction, and image display. Chapter 11 looks at the image characteristics of SPECT, including noise, attenuation, scatter, patient motion, partial-volume effect, and contrast, concluding with the effects of acquisition parameters and filtered backprojection. Chapter 12 covers attenuation correction, scatter compensation, and resolution recovery in SPECT. Once again, the author briefly explains each, weaving in aspects of modern reconstruction techniques to explain how to achieve the best possible resolution of images. Chapter 13 covers quality control and artifacts in SPECT. Table 13-2 is particularly useful in 
that it covers recommended quality control procedures for SPECT and their manufacturer-recommended frequency. Center of rotation is explained, and detailed information on its importance is provided. Other aspects of image development and resolution are covered, including the reference scan, rotational stability, pixel size determination, collimator-hole angulation, and system volume sensitivity. The end of the chapter concentrates on artifacts, which in SPECT include the ray, ring, motion, and truncation types.

Chapters 14 through 17 provide a thorough review of PET, including history, physics, crystal selection, data collection, types of events, time of flight, quantitative ability, tomography composition, detector blocks, acquisition display, image display, energy discrimination, 2-dimensional versus 3-dimensional techniques, performance measures, quality control, and artifacts. Chapter 18 concludes the book with a look at CT as it relates to nuclear medicine. Beginning with the basics of $\mathrm{x}$-ray production, the chapter continues with practical aspects of CT acquisition, reconstruction, and image display. Quality control of CT, as well as image quality and patient dose and public health concerns, is briefly covered, as is radiation protection for the technologist. The chapter ends with a brief description of SPECT/CT and PET/CT technologies.

Overall, this text is a great compilation of information on nuclear instrumentation and a fantastic basic guide to how radiation detectors and imaging techniques work. The comprehensive look at $\gamma$-camera image production and at all aspects of radiation detection makes this book a valuable resource for entry-level technologists. There are many useful tables on troubleshooting common problems and their possible causes. The only thing that could be added to make it even better would be a broader look at filtering and image reconstruction. In the future, a section on MRI could also be added as the possibility of SPECT/MRI and PET/MRI technologies emerge and evolve in our field.

Norman E. Bolus University of Alabama at Birmingham 1705 University Blvd., SHBP, Room 446

Birmingham, AL 35294

E-mail: bolusn@uab.edu 